\title{
Active surveillance for prostate cancer
}

\author{
Daniela K. Shill ${ }^{1}$, Monique J. Roobol ${ }^{2}$, Behfar Ehdaie ${ }^{3}$, Andrew J. Vickers ${ }^{4}$, Sigrid V. Carlsson ${ }^{3,4,5}$ \\ ${ }^{1}$ Hunter College, New York, NY, USA; ${ }^{2}$ Department of Urology, Erasmus University Medical Center, Rotterdam, The Netherlands; ${ }^{3}$ Department \\ of Surgery (Urology Service), Memorial Sloan Kettering Cancer Center, New York, NY, USA; ${ }^{4}$ Department of Epidemiology and Biostatistics, \\ Memorial Sloan Kettering Cancer Center, New York, NY, USA; ${ }^{5}$ Department of Urology, Institute of Clinical Sciences, Sahlgrenska Academy at \\ University of Gothenburg, Sweden \\ Contributions: (I) Conception and design: DK Shill, MJ Roobol, SV Carlsson; (II) Administrative support: None; (III) Provision of study materials \\ or patients: None; (IV) Collection and assembly of data: DK Shill, SV Carlsson, AJ Vickers; (V) Data analysis and interpretation: All authors; (VI) \\ Manuscript writing: All authors; (VII) Final approval of manuscript: All authors. \\ Correspondence to: Sigrid V. Carlsson. 485 Lexington avenue, 10017, New York, NY, USA. Email: carlssos@mskcc.org.
}

\begin{abstract}
Many men diagnosed with localized prostate cancer can postpone definitive treatment without raising their risk of metastasis or death from disease. Active surveillance (AS) is a method of monitoring select men, with the option of switching to active treatment upon signs of progression, thereby avoiding the well-known side-effects of surgery and radiotherapy. This review analyzes the data from long-running AS cohorts to determine the safety and efficacy of AS. We conducted a narrative review of recently published data, including 14 articles from 13 AS cohorts. The cohorts used varying inclusion criteria, with reported differences in clinical T stage and Gleason Score (Grade Group), among other features. Some studies ( $\mathrm{n}=5$ ) limited their cohorts to low-risk patients, while others $(n=8)$ also included intermediate-risk patients. The heterogeneity of the cohorts produced mixed results, with the risk of prostate cancer metastasis ranging from $0.1-1.0 \%$ at 10 years and the risk of prostate cancer mortality ranging from $0-1.9 \%$ at 10 years. However, the majority of studies reported risks of less than $0.5 \%$ at 10 years for both metastasis and death. For most cohorts, half of men remained untreated for 5-10 years, with estimates ranging from $37 \%$ receiving active treatment in the Toronto cohort to $73 \%$ in the Prostate Cancer Research International AS (PRIAS) study. Current data do not support the use of negative magnetic resonance imaging (MRI) to avoid scheduled biopsy. Taken together, the data collected from these AS cohorts suggests that AS is a safe approach for men with low-grade prostate cancer and some men with intermediate risk disease. AS should be more broadly implemented for eligible patients to avoid the decreases in quality of life from undergoing active treatment. Studies expanding the inclusion criteria and further defining a subset of men with favorable intermediate-risk prostate cancer who might safely benefit from AS are needed to assess the long-term outcomes of using AS in intermediate-risk groups.
\end{abstract}

Keywords: Active surveillance (AS); expectant management; watchful waiting; prostate cancer; cohort; program

Submitted Oct 26, 2020. Accepted for publication Mar 25, 2021.

doi: $10.21037 /$ tau-20-1370

View this article at: http://dx.doi.org/10.21037/tau-20-1370

\section{Introduction}

Prostate cancer is currently the second most common cancer diagnosis among men worldwide, with an estimated close to 1.3 million cases in 2018 , expected to nearly double to 2.3 million cases by 2040 (1). Death due to prostate cancer is also common, representing the sixth leading cause of cancer death among men and causing an estimated 359,000 deaths in 2018. As the male population ages and growths, these numbers are expected to increase to 740,000 deaths by 2040 (1).

Early diagnosis of prostate cancer can increase the chances of effective monitoring and treatment, while diagnosis at later stages when the cancer is beyond the window of cure, 
or has metastasized, is more likely to lead to death $(2,3)$. Early diagnosis rates have dramatically increased with the widespread use of prostate specific antigen (PSA) screening, including many slow-growing, indolent prostate cancers, which pose minimal threat and do not have to lead to treatment upfront. Given the psychological and physical tolls of prostate cancer treatment, finding alternative methods for men with low-risk, localized prostate cancer diagnoses can help to lower the burden of disease and improve quality of life for affected men (4).

Active surveillance (AS) is an approach that uses a combination of PSA testing, digital rectal examinations (DRE), and prostate biopsies to monitor prostate cancer in men rather than immediately advising treatment for low-risk/favorable-intermediate risk, localized prostate cancers (5-7). Some AS programs also incorporate magnetic resonance imaging (MRI) at baseline and as part of the follow-up protocol. Using AS can help delay or forgo treatment for these men by monitoring the cancer closely for changes and adjusting accordingly, therefore mitigating overtreatment. Curative therapy for prostate cancer, surgery and radiotherapy, often result in detrimental side effects that can severely impact quality of life, including urinary incontinence, sexual dysfunction, and bowel dysfunction. The potential benefits of AS therefore include the preservation of sexual, urinary, and bowel functions in afflicted men (8). While the proportion of US men with low risk prostate cancer choosing AS was, for many years, only about $10 \%$, this figure is steadily increasing and is now closer to $50 \%$ (9).

The aim of this paper is to review AS as an alternative to immediate active treatment in men with low-risk and favorable-intermediate risk, localized prostate cancer across different cohort studies. The American Urological Association (AUA) recommends "AS as the best available care option for very low risk localized prostate cancer patients" and "as the preferable care option for most low risk localized prostate cancer patients". Regarding intermediate risk, the AUA acknowledges that "AS may be offered to select patients with favorable intermediate risk localized prostate cancer; however, patients should be informed that this comes with a higher risk of developing metastases compared to definitive treatment" (10). If AS is now the preferred option for the initial management of most men with localized, very low-risk and low-risk prostate cancer, current guidelines including the American Society of Clinical Oncology (ASCO) guidelines have supported that select men with low-volume Gleason $3+4$
(Grade Group 2) prostate cancer (intermediate risk) may be considered for AS as well (11). Intermediate risk prostate cancer is indeed a broad, heterogeneous group. This review also seeks to identify which patients are the most clinically appropriate candidates for AS by examining various metrics of completion, notably the percentage of patients who progressed to treatment, the rates of metastasis, and the mortality rates of patients within the cohort studies.

\section{Search strategy}

This review focused on updating the findings of 13 wellestablished and long-running AS cohorts that were previously identified in a review paper conducted by Kinsella et al. in 2018 (7). These cohorts were located at the following institutions: Memorial Sloan Kettering Cancer Center (MSK), The Johns Hopkins University School of Medicine (Johns Hopkins), University of California-San Francisco Helen Diller Family Comprehensive Cancer Center (UCSF), Erasmus University Medical Center (PRIAS), The Miller School of Medicine (University of Miami), The Royal Marsden Hospital (Royal Marsden), The Nuffield Department of Surgical Sciences (ProtecT), University of Toronto Sunnybrook Hospital (Toronto), Copenhagen Prostate Cancer Center (University of Copenhagen), St. Vincent's Prostate Cancer Centre (St. Vincent's Australia), The Sahlgrenska University Hospital (Goteborg), Fred Hutchinson Cancer Research Center (Canary PASS), and the Fondazione IRCCS Istituto Nazionale Tumori Milan (Milan).

Updates on these studies were identified through a systematic search of the Scopus database conducted on July 1, 2020, limited to publications after 2016. The search used the unique IDs of the authors and cohort names, as well as the following keywords to identify studies focused on AS: "active surveillance", "expectant management", "watchful waiting”, "prostate AND cancer", "follow-up”, “cohort", "program", and "programme".

Results from this search strategy were then screened by two authors independently (DKS and SVC) for inclusion in this review. A hand and cited reference search was conducted to retrieve additional updated articles.

\section{Findings}

We first summarize the findings for the 13 included AS cohorts. We then describe current literature and evidence regarding MRI, biomarkers, studies on AS for men with 
intermediate risk prostate cancer and quality of life on AS.

\section{Active surveillance cohorts}

\section{Cobort selection}

This search strategy yielded 418 results, which were screened for eligibility for this review based on their relevance to the 13 AS cohorts of interest. The final review included 14 articles for 13 cohorts in full-text (12-25) (https://cdn. amegroups.cn/static/public/tau-20-1370-1.pdf). These cohorts were dispersed around the world, with 6 cohorts located in North America (12-15,20,22,25), 5 located in Europe $(16,17,19,21,24), 1$ located in Australia (23), and 1 based in multiple countries (18).

\section{Cohort demographics}

The cohorts chosen represented a wide range of patient demographics, as well as a wide range of inclusion criteria that created heterogeneity across the studies. The median patient age ranged from 62 years [Protec $T$ (17)] to 68 years [University of Toronto (22)], and the number of participants ranged from 219 [MSK (13)] to 5,302 [PRIAS (18)]. The median follow-up ranged from 2.3 years to 10 years.

The cohorts evaluated in this review used varying sets of inclusion criteria to determine patient eligibility for AS. Some cohorts had highly restrictive criteria and enrolled only low-risk patients, while others created mixedrisk cohorts by enrolling patients with a wider range of clinical characteristics. Of the 13 cohorts, 5 exclusively enrolled low-risk patients, while 8 included low-risk and intermediate risk-patients.

\section{Inclusion criteria}

Of the studies enrolling only low-risk patients, those with the most restrictive criteria mainly allowed only very-low-risk patients with a maximum clinical $\mathrm{T}$ stage of $\mathrm{T} 2 \mathrm{~A}$, a maximum PSA level of $10 \mathrm{ng} / \mathrm{mL}$, a maximum of Grade Group 1, no more than $2-3$ cores positive, and no more than $50 \%$ cancer involvement in any core [Johns Hopkins (12), University of Copenhagen (21), and Milan (16)]. Others were less restrictive, allowing patients with a maximum clinical $\mathrm{T}$ stage of T2B [Royal Marsden (24) and St. Vincent's Australia (23)] and increasing the maximum PSA level to $15 \mathrm{ng} / \mathrm{mL}$ and Grade Group criteria to Grade Group 2 for patients older than 65 [Royal Marsden (24)].
The rest of the studies included a mixed range of patients. Most cohorts did not exceed a maximum clinical T stage of T2C [MSK (14), UCSF (15), PRIAS (18), University of Miami (25), ProtecT (17), University of Toronto (22), Goteborg (19), and Canary PASS (20)]. The intermediate-risk cohort at MSK similarly recruited patients with a maximum clinical T stage of T2C (2 patients had T3A) (13). There was a lack of consensus around maximum PSA levels for these mixed-risk groups, with some studies suggesting limits of $10 \mathrm{ng} / \mathrm{mL}(18,25)$ and others suggesting a $20 \mathrm{ng} / \mathrm{mL}$ limit $(17,19,20,22)$. Several of the studies had no identified PSA maximum in their reports (13-15).

Most mixed-risk cohorts also recruited patients with a maximum Grade Group score of GG2 [MSK (13), UCSF (15), PRIAS (18), University of Miami (25), University of Toronto (22), Goteborg (19), and Canary PASS (20)]. The ProtecT cohort had no Grade Group restrictions, enrolling patients up to Grade Group 5 (17).

Many of the studies had no reported restrictions of the number of positive biopsy cores in their patients. Of the cohorts with established limits, most restricted to 2 or 3 positive cores [MSK (13), Johns Hopkins (12), University of Miami (25), and University of Copenhagen (21)]. Others restricted based on the percentage of positive biopsy cores out of the total taken, with St. Vincent's Australia (23) limiting to less than 20\%, Milan (16) to less than $25 \%$, and Royal Marsden (24) to less than 50\%.

\section{Follow-up regimen and intervention criteria}

Details of the AS follow-up regimens of these cohorts have been described elsewhere (7,12-26). In brief, AS protocols are variable and heterogeneous across cohorts. Most cohorts utilized regular monitoring with PSA (e.g., every 3-6 months), DRE (e.g., every 6-12 months), confirmatory biopsy (e.g., within 1-1.5 years), and follow-up biopsy (e.g., at 1-3-year intervals). In recent years, several cohorts have included regular MRI (e.g., every 1-3 years). Some protocols employed less intense monitoring (e.g., PSA every 3 months and biopsy every 3-4 years) whereas others used more intense surveillance (e.g., PSA and DRE every 612 months, MRI and re-biopsy every $1-3$ years).

The wide variety of AS protocols and various reasons for switching to radical treatment has been documented previously $(7,26-28)$. The main reason for conversion to active treatment is reclassification; upgrading to Grade Group 2 or higher, $>2$ cores or $>50 \%$ of any core involved are frequently utilized triggers. Some cohorts also initially 
used PSA velocity or PSA doubling time cut-offs to trigger intervention although this has generally changed to using PSA kinetics to prompt further clinical investigation. Patient choice or anxiety are reasons for up to $20 \%$ of patients $(27,28)$.

\section{Patient outcomes}

The outcomes of interest evaluated in this review were the risk of prostate cancer metastasis, the risk of prostate cancer death, and the risk of switching to active treatment. Variations in inclusion or exclusion criteria, methods of reporting risk estimates, length of follow-up, AS protocols, and triggers for intervention created differences in outcomes across the 13 cohorts.

\section{Prostate cancer metastasis}

The risk of prostate cancer metastasis was low across all studies, with many cohorts reporting no patients with distant metastases within 5-10 years [University of Miami (cohort size $\mathrm{N}=230$ ) (25), St. Vincent's Australia $(\mathrm{N}=650)$ (23), Canary PASS ( $\mathrm{N}=905)$ (20), and Milan ( $\mathrm{N}=818)(16)]$, while some cohorts did not report any data on risk of metastatic disease [Royal Marsden ( $\mathrm{N}=471)$ (24) and University of Copenhagen $(\mathrm{N}=317)(21)]$. Two additional cohorts also reported no estimates for risk of distant metastases, although one of those cohorts reported 2 patients with lymph node metastatic disease [MSK $(\mathrm{N}=219)(13)]$, while the other cohort had 4 patients with bone metastatic disease [UCSF $(\mathrm{N}=1,916)(15)]$. Other cohorts similarly reported the crude number of distant metastases in patients, ranging from 8 out of 5,302 patients in the PRIAS study (18) to 33 out of 545 patients in the ProtecT study (17). It should be noted, however, that the cohort of patients enrolled in the monitoring arm of the ProtecT trial does not mimic the cohort of men carefully selected for contemporary AS programs, and as such, the estimate from the Protec $T$ trial is likely an overestimate. The remaining cohorts reported their data as risk estimates for distant metastases, ranging from $0.1 \%$ (95\% CI: $0.04-0.6 \%)$ at 10 years [Johns Hopkins $(\mathrm{N}=1,818)(12)]$ to $1.0 \%(95 \% \mathrm{CI}: 0-4 \%)$ at 10 years [Goteborg ( $\mathrm{N}=474)(19)$ ] and from $0.1 \%$ (95\% CI: $0.04-$ $0.6 \%)$ at 15 years (12) to $7.0 \%(3-16 \%)$ at 15 years (19).

\section{Prostate cancer mortality}

The risk of prostate cancer death in the AS cohorts was similarly low, with many reporting no deaths or a risk of $0 \%$ at 7.5 years and longer [MSK $(13,14)$, University of Miami (25), University of Copenhagen (21), St. Vincent's Australia (23), Canary PASS (20), and Milan (16)]. Reported prostate cancer deaths ranged from 1 out of 5302 patients in the PRIAS cohort (18) to 15 out of 993 patients in the University of Toronto study (22). The estimated risk of prostate cancer death, with $95 \%$ confidence intervals, ranged from $0 \%$ at 10 years [MSK (14)] to $1.2 \%$ at 10 years [ProtecT (17)] and from $0.1 \%$ at 15 years [Johns Hopkins (12)] to $4.0 \%$ at 15 years [Goteborg (19)].

A previous meta-analysis conducted by Enikeev et al. evaluated prostate cancer outcomes in 17 cohorts, comparing AS in low-risk and intermediate-risk patients. The results from their meta-analysis corroborated our findings in this review of 13 cohorts, showing that outcomes of AS vary greatly by risk group and clinical characteristics, making appropriate patient selection a priority (29). The outcomes among very-low-risk and low-risk patients support that AS is a safe strategy for those patients in both the intermediateand long-term (https://cdn.amegroups.cn/static/public/tau20-1370-1.pdf).

\section{Conversion to active treatment}

The risk of converting to active treatment varied depending on the intervention thresholds that were used by the individual institutions. About half of the patients remained untreated for 5 to 10 years. The risk of switching to active treatment over time ranged from $24 \%$ at 5 years [MSK (14)] to $52 \%$ at 5 years [PRIAS (18)] and from $36 \%$ at 10 years (14) to $73 \%$ at 10 years (18). The cohorts that reported shorter follow-up times saw a probability of active treatment between $11 \%$ at 2 years [Royal Marsden (24)] and 29\% at 2 years [Milan (16)]. The longest-running cohort, located at University of Toronto, saw a risk of $45 \%$ at 20 years for conversion to active treatment (22). This creates a range of treatment-free survival across the different AS cohorts, with a majority of patients postponing any form of active treatment for at least 5 years after initial diagnosis.

\section{MRI, biomarkers, intermediate-risk studies, quality of life}

\section{Prostate MRI in active surveillance protocols}

MRI is used in some AS protocols. MRI is frequently used at the start of AS, alongside physical exams and 
confirmatory biopsy, to confirm AS eligibility and to scan for the presence of undetected high-grade and large tumors in the prostate that might have previously been overlooked. MRI is also used throughout AS protocols as a means of monitoring the risk of progression for patients undergoing AS. Current urology guidelines [including e.g., National Comprehensive Cancer Network (NCCN) (30) and American Urological Association (AUA) (31)] emphasize considerations of utilizing MRI at some point during AS to improve the diagnostic specificity, particularly at the start of AS. MRI can be an effective supplement to traditional confirmatory and surveillance biopsies, allowing for more targeted biopsies compared to systematic biopsies alone $(26,32,33)$. Recent studies have investigated the use of MRI as an eventual replacement for surveillance biopsies in specific patient populations. The increased use of MRI has the potential to mitigate the unwanted consequences of repeat biopsies, such as patient anxiety, discomfort, and infectious complications.

Whether MRI can be used to replace AS follow-up biopsy for some patients remains controversial. A study by Stavrinides et al. in 2020 claimed that an MRI-led AS cohort, where monitoring and interventions were guided using MRI results rather than systematic biopsies, had similar outcomes in rates of AS "discontinuation, mortality and metastasis" compared to traditional AS cohorts (34). However, the rates of metastasis were relatively high, 4 cases out of 300 men with Grade Group 2 disease, despite relatively short follow-up ( $\sim 5$ years), and while confidence intervals for rates of metastasis were not presented, they are unlikely to exclude unacceptable risks of metastasis.

A second paper apparently supporting the MRI approach is that of Giganti et al., who studied a UK cohort of 553 patients on AS for low- and intermediate-risk prostate cancer (up to Gleason Score 3+4) using the PRECISE recommendations for MRI stratification. They observed that patients without radiological progression on MRI (PRECISE 1-3) during AS had a very low likelihood of progression on biopsy. However, patients were biopsied based on MRI findings, and hence-as acknowledged by the authors-there is a high risk of verification bias with this study design: if patients without positive MRI findings are not biopsied, these patients will not have adverse findings, and it will appear, in the short term at least, as though negative MRI means no progression (35).

A secondary analysis of a recent randomized controlled trial conducted by Klotz et al. in 2020-the AS MRI (ASIST) study—suggested that performing a baseline MRI before confirmatory biopsy resulted in 50\% fewer AS failures and less grade progression over 2 years compared to the biopsy alone (36). The ASIST study also provided evidence of varying positive predictive value for detecting clinically significant prostate cancer between the participating study sites, highlighting the importance of high-quality MRI, experienced radiologists and high-quality targeted biopsy to maximize utility (37).

However, several studies have found that MRI alone is not an effective substitute for systematic biopsies and raise concerns that the Giganti et al. study missed aggressive cancers in men who were not biopsied on the basis of MRI findings. A study by Liss et al. in 2020 concluded that systematic biopsies should continue to be used for patients, regardless of their MRI results (38). A systematic review by Schoots et al. in 2018 found that MRI-targeted biopsies alone would miss $10 \%$ of cancer grade progressions, while systematic biopsies alone would miss $7 \%$ of cancer grade progressions on AS, emphasizing the need to use both to maximize the detection of potentially aggressive cancers (39). An AS cohort evaluated by Chesnut et al. in 2020 showed that replacing biopsies with MRI can result in missing Grade Group 2 or higher disease in 169 of 1,000 patients, exposing too many patients at risk of undetected disease progression (5). Taken together, the current evidence suggests that the greatest utility of MRI and targeted biopsies comes from its use in conjunction with systematic biopsies in AS programs, rather than in its use as a direct replacement. In particular, a negative MRI should not be used to forgo a scheduled surveillance biopsy at the current time.

\section{Biomarkers for prostate cancer in active surveillance}

The use of biomarkers has expanded in recent years as an additional method for refining risk stratification in select patient populations. In particular, identifying mutations in the BRCA2 gene can help prevent adverse outcomes. BRCA2 gene mutations have been associated with worse outcomes on AS, prompting the need for closer monitoring for these at-risk patients when enrolled in AS cohorts (40).

Studies have evaluated the short-term effects of using tissue-based molecular biomarkers [Oncotype DX Genomic Prostate Score (GPS), Prolaris Cell Cycle Progression (CCP) score, GenomeDx Decipher score, and ProMark] alongside routine clinical indicators, although prospective long-term outcomes of using biomarkers in AS populations have not been evaluated (40). Data suggests that these 
tissue-based assays can be utilized in prostate cancer patients with higher volume Grade Group 1 or favorable intermediate risk Grade Group 2 to help guide clinical decision making and provide more data for determining a management protocol $(40,41)$. However, at the present time, tissue-based biomarkers have not been recommended for routine use and should not be used for patients who have clear clinical indications for either an AS protocol or immediate active treatment (40).

PSA kinetics offers little predictive value and is not being utilized as an independent trigger for intervention. However, PSA density has proven to be useful, with a higher PSA density associated with an increased risk for biopsy reclassification, allowing it to be used as a threshold for inclusion in AS cohorts (41). Urinary markers (PCA3, TMPRSS2:ERG, ExoDx, SelectMDx) have also been evaluated, although the data is largely inconclusive across different studies and needs further validation (41-43). In the prospective multi-institutional Canary PASS study, PCA3 was associated with cancer reclassification in the first surveillance biopsy, but had minimal improvement over clinical variables (44). Both the Prostate Health Index (PHI) and 4 Kscore blood biomarkers have been shown to be associated with improved prediction of clinically significant prostate cancer (42). In the PASS study, addition of the $4 \mathrm{~K}$ panel (free-, total-, intact- PSA and hk2) to a clinical model including variables such as prostate volume and number of prior biopsies improved predictions of high-grade prostate cancer in the first biopsy after diagnosis above and beyond clinical information, however the $4 \mathrm{~K}$ panel did not add value at subsequent surveillance biopsies (45). In the Johns Hopkins AS cohort, baseline and serial measurements of PHI was associated with biopsy reclassification during AS, although the authors did not test whether the marker added to a predictive model including clinical variables (46).

Detailed pathology may also be informative to help determine risk. There is growing evidence that presence of invasive cribriform and/or intraductal carcinoma portend a poorer prognosis, and have therefore been proposed as exclusion criteria for AS (47). However, this remains unproven.

\section{Intermediate-risk studies}

In the past, there has been a reluctance to enroll intermediaterisk patients into AS cohorts as the presence of pattern 4 disease has been an indication for active treatment. However, recent studies have shown that carefully-selected men with favorable intermediate-risk disease could benefit from AS protocols, as long as their cancer remains stable (48). Parameters for careful selection have not been clearly defined across the current guidelines, making it difficult to accurately assess which patients would benefit the most from postponing active treatment. In analyzing data from several randomized control trials comparing immediate treatment to conservative treatment [Scandinavian Prostate Cancer Group-4 (SPCG-4), Prostate Cancer Intervention Versus Observation Trial (PIVOT), and Prostate Testing for Cancer and Treatment (Protec $\mathrm{T})]$, Klotz suggested that candidates for AS among intermediate-risk patients (GG2) should have a low percentage of Gleason pattern 4 and a low PSA density, and would be required to be carefully monitored to evaluate for signs of progression $(48,49)$. Klotz also recommends strict guidelines that include the use of MRI in men with GG2, with $\leq 5-10 \%$ Gleason pattern 4 and either a negative MRI or a positive MRI followed by a favorable targeted biopsy before undergoing an AS protocol (48). The use of genetic risk factors [e.g., BRCA 1/2 gene mutations, HOXB13 gene mutation, DNAgene repair mutations (ATM, CHEK2, MSH2), and singlenucleotide polymorphisms] can also be used to identify appropriate AS candidates among intermediate-risk patients, as a significant proportion of AS failures might be caused by undetected genetic predispositions towards more aggressive cancers (48).

A meta-analysis showed that AS was a viable first management option for carefully selected and monitored men with intermediate-risk prostate cancer. AS for select intermediate-risk prostate cancer patients showed beneficial results in the short-term, with similar rates of treatment as well as adverse oncologic outcome at 5 years across both low-risk and intermediate-risk groups following AS protocols (29). On longer term follow-up at 10 or 15 years, there are higher although still relatively low rates of metastasis in intermediate risk patients (29). The use of MRI as part of a comprehensive risk assessment at baseline of AS can also be helpful in facilitating the selection of appropriate intermediate risk patients for AS. Even though the studies in the meta-analysis, the Klotz review, and our review all had varying inclusion criteria and often used different AS protocols, these combined results suggest that AS is a safe option for postponing treatment and could be used in specific groups of low-intermediate-risk prostate cancer patients, particularly in the first few years post diagnosis.

Given the higher rates of adverse outcomes in intermediaterisk patients after 10 or more years on AS, it could be 
preferable to employ more stringent monitoring, particularly in later years, and potentially consider switching intermediaterisk patients to active treatment at higher rates than their low-risk counterparts. Using data from genetic markers can also guide these decisions and decrease the risk of missing the opportunity to administer effective treatment (48). Intermediate-risk prostate cancer patients can benefit from a wide range of potential interventions, including radiotherapy and radical prostatectomy, but AS should also be offered as an option for patients according to their clinical presentation, including consideration of total length of Gleason pattern 4 and personal preferences (50).

\section{Quality of life under active surveillance}

Some physicians have hesitated to recommend AS to their patients out of a fear of invoking anxiety, elevating stress, and causing other adverse mental health outcomes $(51,52)$. Selfevaluations from prostate cancer patients undergoing AS show that AS patients assess their quality of life positively and show high ratings across all health state utilities (51). In the randomized Protec T trial, comparing radical prostatectomy, radiotherapy and active monitoring, there were no significant between group differences in anxiety at any time or in cancer-related quality of life at 5 years of follow-up (53). Indeed, a systematic review of 34 studies (12,497 patients) suggested that rates of anxiety and depression in AS groups are either lower or equivalent to rates in population control groups, showing that remaining on AS does not negatively impact mental health (54). A study looking more specifically at prostate cancer-related anxiety included 413 men on AS. It was common for men to report mild prostate cancer-specific anxiety after the initiation of AS (29\% during the first year), however anxiety decreased significantly with time as most patients adjusted, and the majority reported low anxiety levels within 2 years (55). In comparison, low psychological wellbeing at 12- and 24-months follow-up in patients undergoing radical prostatectomy has been reported, emphasizing the need for broader availability of psychological resources for patients undergoing active treatment (52).

Additionally, due to the regimented follow-up procedures part of AS protocols, patients in AS programs feel increased control over their cancer rather than uncertainty, helping to minimize cancer-related fears and anxieties (56). This increased control also comes from the decision-making process, which allows patients to have autonomy over their management protocol and improves patient well-being (57). AS protocols that consider patient preferences and allow this collaboration between patients and physicians can help to reduce adverse effects on quality of life $(4,57)$.

\section{Future directions}

The Movember Foundation's Global Action Plan Prostate Cancer Active Surveillance (GAP3) cohort (58) will the discoveries of the last 5 years change the future?" by MJR in this series of TAU-will continue to expand its database with additional centers, increasing follow-up, and also with additional parameters (e.g., imaging modalities, biomarkers, genomics, and follow-up after AS including biochemical recurrence after treatment, risk of metastasis and survival). Currently, the GAP3 cohort includes over 21,000 men from 28 cohorts, of which over 1,000 patients have at least 10 years follow-up. On the basis of this comprehensive dataset, critical research questions in AS will be addressed focusing on reducing the burden of being on AS while maintaining safety.

Ongoing efforts are focused on tailoring AS to individual patient needs and disease characteristics, thus aiming to design dynamic risk-based AS strategies that ensures safety, improves the experience of AS and lessens the patient burden of intensive monitoring. Research investigating whether MRI and/or biomarkers can replace surveillance biopsy is ongoing $(33,59)$. Risk calculators such as the Canary Prostate Active Surveillance Study Risk Calculators (PASS-RCs) are also being studied to help predict reclassification on biopsy (60). A recent modeling study accounting for variation in AS protocols between four large AS cohorts suggested that biennial biopsies could be an alternative to annual biopsies (61). An ongoing largescale randomized trial, Prostate Cancer Active Surveillance Trigger trial (PCASTt/SPCG-17), will shed further light on how to optimize current AS protcols by comparing current clinical care to an AS protocol with standardized triggers based on MRI and PSA density to elicit repeat biopsies and MRI and histopathological progression to trigger radical treatment (62).

\section{Conclusions}

Current data are clear that AS should be the standard of care in patients with pattern 3 disease (Grade Group 1) and can be considered in carefully selected men with low volume favorable intermediate risk disease. Future studies should focus on further refining the eligibility for AS and triggers for active treatment. Many of the guidelines in place 
currently use varying inclusion criteria, and it is likely that many men who could benefit from AS are excluded. Using MRI and biomarkers to guide the selection of intermediaterisk patients suitable for AS can allow many men to benefit from avoidance or delay of curative therapy without risking progression of their disease to an aggressive form. Finally, studies focusing on improving our understanding of factors that influence the acceptance, adherence to and discontinuation of AS across various populations worldwide should be encouraged.

\section{Acknowledgments}

We sincerely thank Research Informationist Lindsay Boyce, MLIS for assistance with the literature search and Dr. Laura Liberman, Office of Faculty Development, Memorial Sloan Kettering Cancer Center, New York, USA, for the 2020 Summer Clinical Oncology Research Experience (SCORE) Program that enabled DKS's work on this study.

Funding: SVC's, AJV's and BE's work on this paper was supported in part by funding from the National Institutes of Health/National Cancer Institute (P30-CA008748) and the Sidney Kimmel Center for Prostate and Urologic Cancers. SVC was further supported by a career development award from the National Institutes of Health/National Cancer Institute (K22-CA234400) and U01-CA1999338-02. The funding bodies had no role in the preparation, review, or approval of the manuscript or the decision to submit the manuscript for publication.

\section{Footnote}

Provenance and Peer Review: This article was commissioned by the Guest Editors (Mieke Van Hemelrijck and Netty Kinsella) for the series "Expectant Management in Genitourinary Malignancies (Prostate, Bladder, Kidney)" published in Translational Andrology and Urology. The article has undergone external peer review.

Conflicts of Interest: The authors have completed the ICMJE uniform disclosure form (available at http:// dx.doi.org/10.21037/tau-20-1370). The series "Expectant Management in Genitourinary Malignancies (Prostate, Bladder, Kidney)" was commissioned by the editorial office without any funding or sponsorship. Dr. Roobol serves as an unpaid Associate Editor-in-Chief of Translational Andrology and Urology from Jan 2020 to Dec 2021. Dr. Ehdaie reports personal fees from Myriad Genetics, personal fees from Koelis, outside the submitted work. Dr. Vickers reports personal fees from Arctic Partners, personal fees from Opko, personal fees from Steba, personal fees from InsightTec, during the conduct of the study; In addition, Dr. Vickers has a patent Arctic Partners issued. The authors have no other conflicts of interest to declare.

Ethical Statement: The authors are accountable for all aspects of the work in ensuring that questions related to the accuracy or integrity of any part of the work are appropriately investigated and resolved.

Open Access Statement: This is an Open Access article distributed in accordance with the Creative Commons Attribution-NonCommercial-NoDerivs 4.0 International License (CC BY-NC-ND 4.0), which permits the noncommercial replication and distribution of the article with the strict proviso that no changes or edits are made and the original work is properly cited (including links to both the formal publication through the relevant DOI and the license). See: https://creativecommons.org/licenses/by-nc-nd/4.0/.

\section{References}

1. Culp MB, Soerjomataram I, Efstathiou JA, et al. Recent Global Patterns in Prostate Cancer Incidence and Mortality Rates. Eur Urol 2020;77:38-52.

2. Hugosson J, Roobol MJ, Mansson M, et al. A 16yr Follow-up of the European Randomized study of Screening for Prostate Cancer. Eur Urol 2019;76:43-51.

3. Shoag JE, Nyame YA, Gulati R, et al. Reconsidering the Trade-offs of Prostate Cancer Screening. N Engl J Med 2020;382:2465-8.

4. Venderbos LDF, Aluwini S, Roobol MJ, et al. Long-term follow-up after active surveillance or curative treatment: quality-of-life outcomes of men with low-risk prostate cancer. Qual Life Res 2017;26:1635-45.

5. Chesnut GT, Vertosick EA, Benfante N, et al. Role of Changes in Magnetic Resonance Imaging or Clinical Stage in Evaluation of Disease Progression for Men with Prostate Cancer on Active Surveillance. Eur Urol 2020;77:501-7.

6. Mottet N, Bellmunt J, Briers E, et al. EAU - ESTRO - ESUR - SIOG Guidelines on Prostate Cancer. Edn. presented at the EAU Annual Congress Amsterdam 2020. 978-94-92671-07-3. Publisher: EAU Guidelines Office. Place published: Arnhem, The Netherlands. Available online: https://uroweb.org/guideline/prostate-cancer/. 
7. Kinsella N, Helleman J, Bruinsma S, et al. Active surveillance for prostate cancer: a systematic review of contemporary worldwide practices. Transl Androl Urol 2018;7:83-97.

8. Hoffman KE, Penson DF, Zhao Z, et al. Patient-Reported Outcomes Through 5 Years for Active Surveillance, Surgery, Brachytherapy, or External Beam Radiation With or Without Androgen Deprivation Therapy for Localized Prostate Cancer. JAMA 2020;323:149-63.

9. Cooperberg MR, Carroll PR. Trends in Management for Patients With Localized Prostate Cancer, 1990-2013. JAMA 2015;314:80-2.

10. Sanda MG, Cadeddu JA, Kirkby E, et al. Clinically Localized Prostate Cancer: AUA/ASTRO/SUO Guideline. Part I: Risk Stratification, Shared Decision Making, and Care Options. J Urol 2018;199:683-90.

11. Chen RC, Rumble RB, Loblaw DA, et al. Active Surveillance for the Management of Localized Prostate Cancer (Cancer Care Ontario Guideline): American Society of Clinical Oncology Clinical Practice Guideline Endorsement. J Clin Oncol 2016;34:2182-90.

12. Tosoian JJ, Mamawala M, Epstein JI, et al. Active Surveillance of Grade Group 1 Prostate Cancer: Longterm Outcomes from a Large Prospective Cohort. Eur Urol 2020;77:675-82.

13. Carlsson S, Benfante N, Alvim R, et al. Risk of Metastasis in Men with Grade Group 2 Prostate Cancer Managed with Active Surveillance at a Tertiary Cancer Center. J Urol 2020;203:1117-21.

14. Carlsson S, Benfante N, Alvim R, et al. Long-Term Outcomes of Active Surveillance for Prostate Cancer: The Memorial Sloan Kettering Cancer Center Experience. J Urol 2020;203:1122-7.

15. Balakrishnan AS, Cowan JE, Cooperberg MR, et al. Evaluating the Safety of Active Surveillance: Outcomes of Deferred Radical Prostatectomy after an Initial Period of Surveillance. J Urol 2019;202:506-10.

16. Marenghi C, Alvisi MF, Palorini F, et al. Elevenyear management of prostate cancer patients on active surveillance: what have we learned? Tumori 2017;103:464-74.

17. Hamdy FC, Donovan JL, Lane JA, et al. 10-Year Outcomes after Monitoring, Surgery, or Radiotherapy for Localized Prostate Cancer. N Engl J Med 2016;375:1415-24.

18. Bokhorst LP, Valdagni R, Rannikko A, et al. A Decade of Active Surveillance in the PRIAS Study: An Update and Evaluation of the Criteria Used to Recommend a Switch to Active Treatment. Eur Urol 2016;70:954-60.

19. Godtman RA, Holmberg E, Khatami A, et al. Longterm Results of Active Surveillance in the Goteborg Randomized, Population-based Prostate Cancer Screening Trial. Eur Urol 2016;70:760-6.

20. Newcomb LF, Thompson IM Jr, Boyer HD, et al. Outcomes of Active Surveillance for Clinically Localized Prostate Cancer in the Prospective, Multi-Institutional Canary PASS Cohort. J Urol 2016;195:313-20.

21. Thomsen FB. Active surveillance strategy for patients with localised prostate cancer: criteria for progression. Dan Med J 2015;62:B5005.

22. Klotz L, Vesprini D, Sethukavalan P, et al. Long-term follow-up of a large active surveillance cohort of patients with prostate cancer. J Clin Oncol 2015;33:272-7.

23. Thompson JE, Hayen A, Landau A, et al. Mediumterm oncological outcomes for extended vs saturation biopsy and transrectal vs transperineal biopsy in active surveillance for prostate cancer. BJU Int 2015;115:884-91.

24. Selvadurai ED, Singhera M, Thomas K, et al. Mediumterm outcomes of active surveillance for localised prostate cancer. Eur Urol 2013;64:981-7.

25. Soloway MS, Soloway CT, Eldefrawy A, et al. Careful selection and close monitoring of low-risk prostate cancer patients on active surveillance minimizes the need for treatment. Eur Urol 2010;58:831-5.

26. Komisarenko M, Martin LJ, Finelli A. Active surveillance review: contemporary selection criteria, follow-up, compliance and outcomes. Transl Androl Urol 2018;7:243-55.

27. Van Hemelrijck M, Ji X, Helleman J, et al. Reasons for Discontinuing Active Surveillance: Assessment of 21 Centres in 12 Countries in the Movember GAP3 Consortium. Eur Urol 2019;75:523-31.

28. Simpkin AJ, Tilling K, Martin RM, et al. Systematic Review and Meta-analysis of Factors Determining Change to Radical Treatment in Active Surveillance for Localized Prostate Cancer. Eur Urol 2015;67:993-1005.

29. Enikeev D, Morozov A, Taratkin M, et al. Active Surveillance for Intermediate-Risk Prostate Cancer: Systematic Review and Meta-analysis of Current Protocols and Outcomes. Clin Genitourin Cancer 2020;18:e739-e753.

30. NCCN Guidelines Prostate Cancer Version 2.2020. Available online: http://www.nccn.org

31. Sanda MG, Cadeddu JA, Kirkby E, et al. Clinically Localized Prostate Cancer: AUA/ASTRO/SUO Guideline. Part II: Recommended Approaches and Details 
of Specific Care Options. J Urol 2018;199:990-7.

32. Perlis N, Klotz L. Contemporary Active Surveillance: Candidate Selection, Follow-up Tools, and Expected Outcomes. Urol Clin North Am 2017;44:565-74.

33. Klotz L. Active surveillance for low-risk prostate cancer. Curr Opin Urol 2017;27:225-30.

34. Stavrinides V, Giganti F, Trock B, et al. Five-year Outcomes of Magnetic Resonance Imaging-based Active Surveillance for Prostate Cancer: A Large Cohort Study. Eur Urol 2020;78:443-51.

35. Giganti F, Stabile A, Stavrinides V, et al. Natural history of prostate cancer on active surveillance: stratification by MRI using the PRECISE recommendations in a UK cohort. Eur Radiol 2021;31:1644-55.

36. Klotz L, Pond G, Loblaw A, et al. Randomized Study of Systematic Biopsy Versus Magnetic Resonance Imaging and Targeted and Systematic Biopsy in Men on Active Surveillance (ASIST): 2-year Postbiopsy Follow-up. Eur Urol 2020;77:311-7.

37. Kasivisvanathan V, Giganti F, Emberton M, et al. Magnetic Resonance Imaging Should Be Used in the Active Surveillance of Patients with Localised Prostate Cancer. Eur Urol 2020;77:318-9.

38. Liss MA, Newcomb LF, Zheng Y, et al. Magnetic Resonance Imaging for the Detection of High Grade Cancer in the Canary Prostate Active Surveillance Study. J Urol 2020;204:701-6.

39. Schoots IG, Nieboer D, Giganti F, et al. Is magnetic resonance imaging-targeted biopsy a useful addition to systematic confirmatory biopsy in men on active surveillance for low-risk prostate cancer? A systematic review and meta-analysis. BJU Int 2018;122:946-58.

40. Eggener SE, Rumble RB, Armstrong AJ, et al. Molecular Biomarkers in Localized Prostate Cancer: ASCO Guideline. J Clin Oncol 2020;38:1474-94.

41. Loeb S, Tosoian JJ. Biomarkers in active surveillance. Transl Androl Urol 2018;7:155-9.

42. Becerra MF, Bhat A, Mouzannar A, et al. Serum and urinary biomarkers for detection and active surveillance of prostate cancer. Curr Opin Urol 2019;29:593-7.

43. Loeb S, Bruinsma SM, Nicholson J, et al. Active surveillance for prostate cancer: a systematic review of clinicopathologic variables and biomarkers for risk stratification. Eur Urol 2015;67:619-26.

44. Newcomb LF, Zheng Y, Faino AV, et al. Performance of PCA3 and TMPRSS2:ERG urinary biomarkers in prediction of biopsy outcome in the Canary Prostate Active Surveillance Study (PASS). Prostate Cancer
Prostatic Dis 2019;22:438-45.

45. Lin DW, Newcomb LF, Brown MD, et al. Evaluating the Four Kallikrein Panel of the 4Kscore for Prediction of High-grade Prostate Cancer in Men in the Canary Prostate Active Surveillance Study. Eur Urol 2017;72:448-54.

46. Tosoian JJ, Loeb S, Feng Z, et al. Association of [-2] proPSA with biopsy reclassification during active surveillance for prostate cancer. J Urol 2012;188:1131-6.

47. Kweldam CF, Kummerlin IP, Nieboer D, et al. Presence of invasive cribriform or intraductal growth at biopsy outperforms percentage grade 4 in predicting outcome of Gleason score 3+4=7 prostate cancer. Mod Pathol 2017;30:1126-32.

48. Klotz L. Active surveillance in intermediate-risk prostate cancer. BJU Int 2020;125:346-54.

49. Klotz L. Active Surveillance for Intermediate Risk Prostate Cancer. Curr Urol Rep 2017;18:80.

50. Preisser F, Cooperberg MR, Crook J, et al. Intermediaterisk Prostate Cancer: Stratification and Management. Eur Urol Oncol 2020;3:270-80.

51. Loeb S, Curnyn C, Walter D, et al. Health state utilities among contemporary prostate cancer patients on active surveillance. Transl Androl Urol 2018;7:197-202.

52. Stinesen Kollberg K, Thorsteinsdottir T, Wilderang U, et al. Social constraints and psychological well-being after prostate cancer: A follow-up at 12 and 24 months after surgery. Psychooncology 2018;27:668-75.

53. Donovan JL, Hamdy FC, Lane JA, et al. Patient-Reported Outcomes after Monitoring, Surgery, or Radiotherapy for Prostate Cancer. N Engl J Med 2016;375:1425-37.

54. Carter G, Clover K, Britton B, et al. Wellbeing during Active Surveillance for localised prostate cancer: a systematic review of psychological morbidity and quality of life. Cancer Treat Rev 2015;41:46-60.

55. Marzouk K, Assel M, Ehdaie B, et al. Long-Term Cancer Specific Anxiety in Men Undergoing Active Surveillance of Prostate Cancer: Findings from a Large Prospective Cohort. J Urol 2018;200:1250-5.

56. Dordoni P, Badenchini F, Alvisi MF, et al. How do prostate cancer patients navigate the active surveillance journey? A 3-year longitudinal study. Support Care Cancer 2021;29:645-51.

57. Menichetti J, Valdagni R, Bellardita L. Quality of life in active surveillance and the associations with decision-making-a literature review. Transl Androl Urol 2018;7:160-9.

58. Bruinsma SM, Zhang L, Roobol MJ, et al. The Movember 
Foundation's GAP3 cohort: a profile of the largest global prostate cancer active surveillance database to date. BJU Int 2018;121:737-44.

59. Leapman MS, Carroll PR. What is the best way not to treat prostate cancer? Urol Oncol 2017;35:42-50.

60. Drost FH, Nieboer D, Morgan TM, et al. Predicting Biopsy Outcomes During Active Surveillance for Prostate Cancer: External Validation of the Canary Prostate Active Surveillance Study Risk Calculators in Five Large Active

Cite this article as: Shill DK, Roobol MJ, Ehdaie B, Vickers AJ, Carlsson SV. Active surveillance for prostate cancer. Transl Androl Urol 2021;10(6):2809-2819. doi: 10.21037/tau-20-1370
Surveillance Cohorts. Eur Urol 2019;76:693-702.

61. Inoue LYT, Lin DW, Newcomb LF, et al. Comparative Analysis of Biopsy Upgrading in Four Prostate Cancer Active Surveillance Cohorts. Ann Intern Med 2018;168:1-9.

62. Ahlberg MS, Adami HO, Beckmann K, et al. PCASTt/ SPCG-17-a randomised trial of active surveillance in prostate cancer: rationale and design. BMJ Open 2019;9:e027860. 